\title{
A retrospective study on the impact of bar flipping on the recurrence of pectus excavatum after the Nuss procedure
}

\author{
Yu-Jiun Fan' ${ }^{1}$, Po-Cheng Lo ${ }^{1}$, Yuan-Yu Hsu' ${ }^{2}$ I-Shiang Tzeng ${ }^{3}$, Bo-Chun Wei ${ }^{1}$ and Yeung-Leung Cheng ${ }^{1,4^{*}}$ (D)
}

\begin{abstract}
Background: The Nuss procedure is widely used to correct pectus excavatum. Bar displacement is a common complication associated with this procedure. How the flipping of the bar affects pectus excavatum recurrence has not been reported. In our study, we discuss this and also offer an easier method to determine bar flipping.

Methods: This retrospective study analyzed pectus excavatum patients who underwent primary Nuss repair from August 2014 to December 2018. The preoperative and postoperative Haller indices were measured on chest radiographs ( $c x r H I)$. The slope angle of bar flipping (a) was measured on lateral chest radiographs. The improvement index after surgical repair was calculated by: ([preoperative $c x r H I$-postoperative $c x r H I] /$ preoperative $c x r H I \times 100$ ). The impact of a on the improvement index was analyzed using one-way analysis of variance and receiver operating characteristic tests.
\end{abstract}

Results: In this study, 359 adult and adolescent patients with an average age of $23.9 \pm 7.7$ years were included. We formed four subgroups based on the a value: $a \leq 10^{\circ}(n=131), a=11-20^{\circ}(n=154), a=21-30^{\circ}(n=51)$, and $a>30^{\circ}(n=23)$. The mean improvement indices in these groups were $27 \%, 28 \%, 26 \%$, and $13 \%$, respectively. Patients with $a>30^{\circ}$ were associated with a significantly poorer improvement index than those from the other subgroups $(p<0.001)$.

Conclusions: The a value is an alternative measurement method for presenting the radiological outcomes after the Nuss procedure. An $a>30^{\circ}$ indicates a possible recurrence of pectus excavatum after the Nuss repair. Surgical revision may be considered in patients with an $a>30^{\circ}$, while monitoring should be considered in the other patient groups.

Keywords: Pectus excavatum, Nuss procedure, Bar flipping, Recurrence

\section{Introduction}

Pectus excavatum (PE), also known as funnel chest, is a structural deformity in which the sternum sinks in the center of the anterior chest wall. The exact cause of PE remains unknown. This congenital problem may become more severe in adolescence, as the chest wall deformation

\footnotetext{
*Correspondence: ndmc0928@yahoo.com.tw

${ }^{1}$ Division of Thoracic Surgery, Department of Surgery, Taipei Tzu Chi Hospital, Buddhist Tzu Chi Medical Foundation, New Taipei City, No. 289, Jianguo Road, Xindian District 231, Taiwan

Full list of author information is available at the end of the article
}

may be aggravated by an overgrowth of the costal cartilage. The estimated prevalence of PE in the adult population is 1 in 250 individuals [1]. Patients with PE do have not only a negative body image but also experience physical problems such as chest pain, exercise intolerance, dyspnea, sleep apnea, and rapid heart rate due to the compressive effects on the heart and lungs [2]. The Nuss procedure, first reported in 1988 by Nuss et al., is a minimally invasive method widely used to correct PE. In this method, metal bars are placed retrosternally, with the lateral ribs acting as hinge points, to lift the depressed chest wall. original author(s) and the source, provide a link to the Creative Commons licence, and indicate if changes were made. The images or other third party material in this article are included in the article's Creative Commons licence, unless indicated otherwise in a credit line to the material. If material is not included in the article's Creative Commons licence and your intended use is not permitted by statutory regulation or exceeds the permitted use, you will need to obtain permission directly from the copyright holder. To view a copy of this licence, visit http://creativecommons.org/licenses/by/4.0/. The Creative Commons Public Domain Dedication waiver (http://creativeco mmons.org/publicdomain/zero/1.0/) applies to the data made available in this article, unless otherwise stated in a credit line to the data. 
Despite the excellent outcomes, a series of outcome studies have revealed that bar migration and PE recurrence are the major problems associated with the Nuss procedure [3-6]. The reported bar migration rates vary [5-7]. Previous investigators have classified the mechanisms of bar migration into bar flipping (bar rotates), lateral sliding (bar slides horizontally to one side), and hinge-point disruption (bar shifts dorsally) [7]. According to our clinical observation, PE recurrence is most likely associated with bar flipping (to some degree) rather than lateral sliding. However, no study has investigated the association between bar flipping and outcomes of the Nuss procedure. Therefore, this study aimed to identify the association between the degree of bar flipping and $\mathrm{PE}$ recurrence. Besides, there is still no standard consensus on the measurement of the bar flipping degree. We designed a method to measure the slope of the bar and to estimate PE recurrence. Our study provides a guideline to survey and follow patients who may warrant revision surgery after the Nuss procedure.

\section{Methods}

Between August 2014 and December 2018, 374 patients underwent a primary repair for PE at the Taipei ChiTzu Hospital in New Taipei City, Taiwan. Of these, 367 patients received the Nuss repair, while seven received the Ravitch repair. Of the 367 patients who underwent the Nuss procedure, 359 of which were adolescents and adults above 12 years of age and they were included in this retrospective analysis (Fig. 1). This retrospective study was approved by the Ethics Committee and the Institutional Review Board of the Taipei Tzu Chi Hospital, Taipei, Taiwan, ROC (IRB No: 08-X-101). Patient consent requirement was waived by the institutional review board due to the study's retrospective nature.

All surgeries were performed by a single surgeon (Dr. Cheng); the surgical technique followed has been described previously [8]. The indications for surgery were based on the criteria established by Nuss and Kelly [9]. Patients' baseline characteristics, including sex, age,

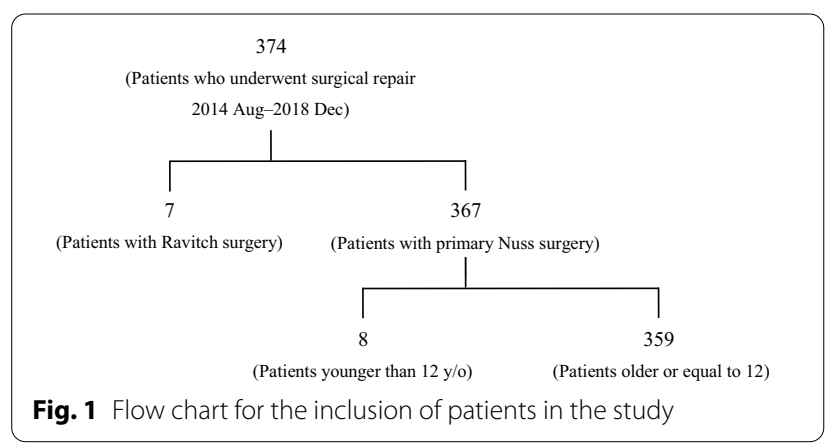

body weight, height, preoperative Haller index, and/or the presence of scoliosis, were recorded as preoperative assessments. Further preoperative examinations included chest radiography, electrocardiography, pulmonary function test, echocardiography, and chest computed tomography $(\mathrm{CT})$. The Haller index $(\mathrm{HI})[10]$, which is the ratio of the maximum transverse thoracic diameter to the minimum sternal-to-anterior vertebral body distance on chest CT, was calculated on anteroposterior and lateral views of chest radiographs instead (cxrHI) (Fig. 1). The preoperative and postoperative cxrHI could be used to estimate the preoperative severity of $\mathrm{PE}$ and postoperative outcomes $[11,12]$. Because poor improvement in $\mathrm{PE}$ after surgery can indicate PE recurrence, we used the change of cxrHI $(\Delta c x r H I)$ (i.e., the improvement index) to estimate the degree of $\mathrm{PE}$ recurrence in an objective way using the following formula: [(preoperative cxrHIpostoperative $c x r \mathrm{HI}) /$ preoperative $c x r \mathrm{HI}] \times 100$.

We also designed an easier method for measuring the degree of bar flipping by using the slope angle $(\alpha)$. On lateral chest radiography, $\alpha$ was measured as the angle between the two lines connecting the midpoint of the two end holes of the bar to 1) the uppermost part of the arch of the bent bar and 2) the expected point of the dorm's optimal position (Fig. 2). The HI and the $\alpha$ on the chest radiograph were measured by an experienced surgeon and a radiologist, who were both blinded to the clinical data.

\section{Statistical analyses}

Statistical analyses were performed using IBM SPSS Statistics for Windows, version 24 (IBM Corp., Armonk, NY, USA). The Kolmogorov-Smirnov test revealed that all investigated parameters in our study were distributed normally. Continuous data were expressed as means \pm standard deviations, while categorical data were expressed as counts (\%). The optimal cuff-off value for the angle of bar flipping was determined by receiver operating characteristic (ROC) curve analysis. Based on the $\alpha$ of the most severe bar flipping, the patients were categorized into four groups, namely, $\alpha \leq 10^{\circ}, \alpha=11-20^{\circ}$, $\alpha=21-30^{\circ}$, and $\alpha>30^{\circ}$. The improvement indices were compared between these groups using one-way analysis of variance (ANOVA), and the Scheffe test was used as a post hoc test. Using the cut-off value at $\alpha=30^{\circ}$, the differences between the groups were analyzed using a two-sample $t$-test for continuous data and the chi-square test for categorical data. ROC curves were created using varying values for $\alpha$ as the categorization criteria and the I index to compare the best area under the curve (AUC) value. The DeLong test was further used to compare the $C$-statistic between different $\alpha$ values (Fig. 3) [13]. ROC curve comparisons were conducted using $R$ version 4.0.0 


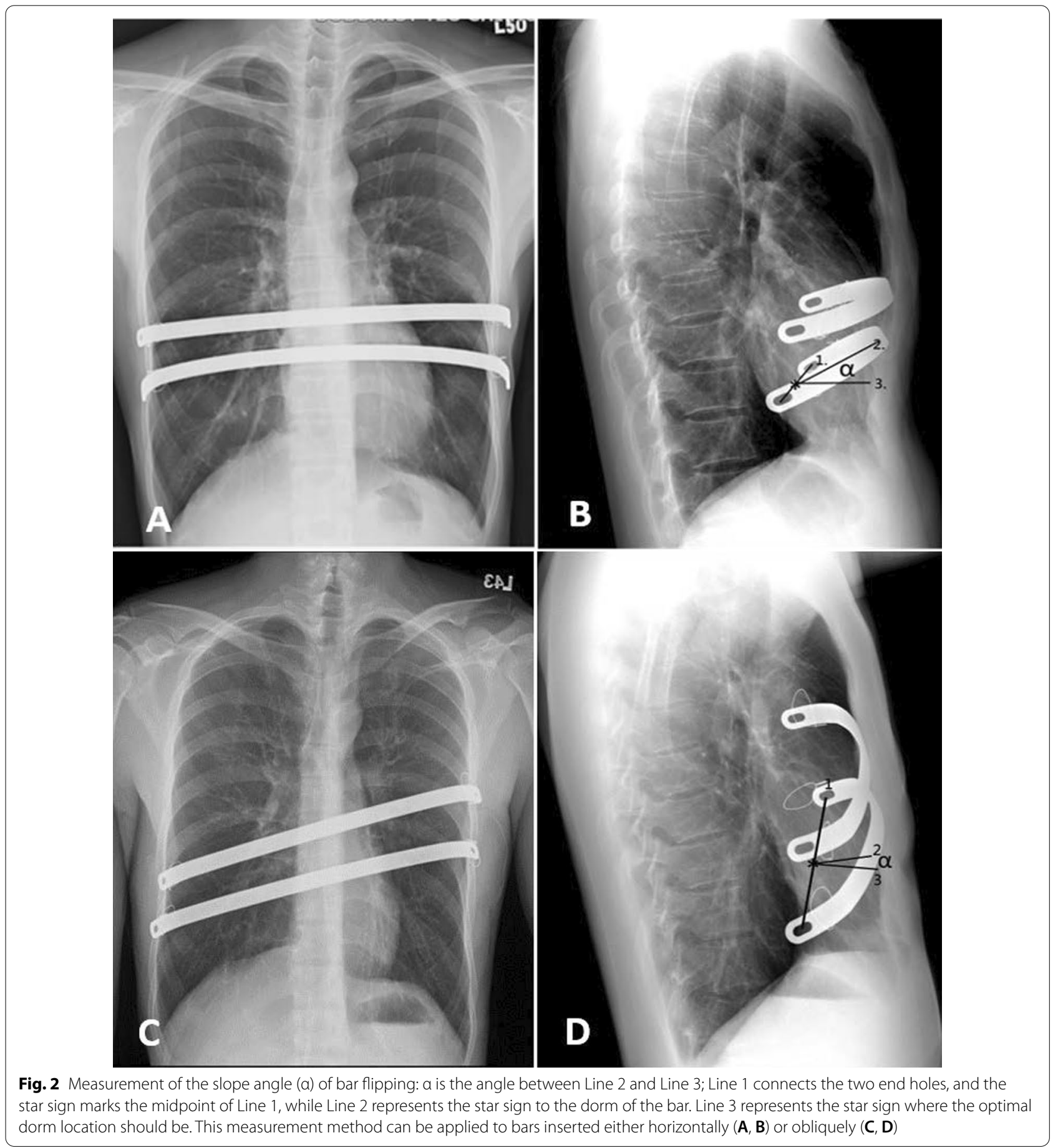

(pROC package). A $p$ value of $<0.05$ was considered statistically significant.

\section{Results}

The study group comprised 304 men and 55 women with an average age of $23.9 \pm 7.7$ years. The mean postoperative follow-up period was $2.2 \pm 1.3$ years, while no individual follow-up was of $<6$ months. The $\alpha \leq 10^{\circ}$, $\alpha=11-20^{\circ}, \alpha=21-30^{\circ}$, and $\alpha>30^{\circ}$ groups comprised $131,154,51$, and 23 patients, respectively (Table 1). The improvement indices in the $\alpha \leq 10^{\circ}, \alpha=11-20^{\circ}$, $\alpha=21-30^{\circ}$, and $\alpha>30^{\circ}$ groups were $27 \%$ [95\% confidence interval (CI): $17-37 \%$ ], $28 \%$ (95\% CI: $16-40 \%$ ), 26\% (95\% CI: $14-38 \%)$, and 13\% (95\% CI: 4-22\%), respectively 


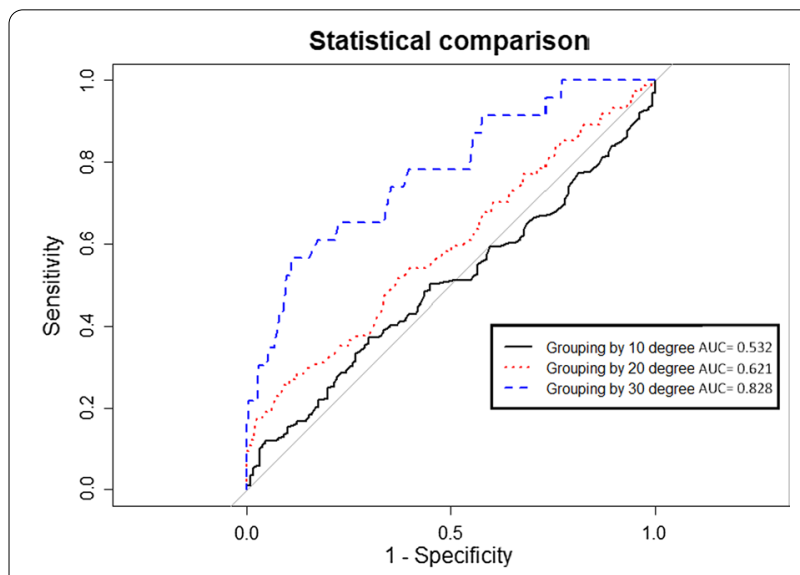

Fig. 3 The DeLong test shows that grouping by $10^{\circ}$ and grouping by $20^{\circ}$ are not significant $(p=0.061)$. Grouping by $20^{\circ}$ and $30^{\circ}(p=0.006)$ and grouping by $10^{\circ}$ and $30^{\circ}(p<0.001)$ are significant

Table 1 Descriptive statistics of the different groups

\begin{tabular}{lrllll}
\hline Flipping angle & $\mathbf{N}$ & cxrHlpre $^{\mathbf{a}}$ & cxrHIpost $^{\mathbf{b}}$ & $\Delta$ cxrHI $^{\mathbf{c}}$ & I index $^{\mathbf{d}}$ \\
\hline$\leq 10^{\circ}$ & 131 & $3.69 \pm 0.7$ & $2.66 \pm 0.4$ & $1.03 \pm 0.57$ & $27 \pm 10$ \\
$11-20^{\circ}$ & 154 & $3.95 \pm 0.8$ & $2.77 \pm 0.4$ & $1.18 \pm 0.77$ & $28 \pm 12$ \\
$21-30^{\circ}$ & 51 & $3.94 \pm 0.9$ & $2.86 \pm 0.4$ & $1.09 \pm 0.7$ & $26 \pm 12$ \\
$>30^{\circ}$ & 23 & $3.96 \pm 1.5$ & $3.46 \pm 1.3$ & $0.5 \pm 0.5$ & $13 \pm 9$ \\
\hline
\end{tabular}

${ }^{a}$ Haller index on the preoperative chest radiograph

${ }^{\mathrm{b}}$ Haller index on the postoperative chest radiograph

'cxrHlpre-cxrHlpost

${ }^{d}$ improvement index $=(\mathrm{cxrHIpre}-\mathrm{cxrHIpost}) / \mathrm{cxrH}$ pre

(Table 1). The higher the improvement index, the more modest was the postoperative PE improvement. Oneway ANOVA and post hoc analysis of the improvement indices within the groups revealed that an $\alpha>30^{\circ}$ was associated with a decreased improvement index, indicating a low clinical improvement $(p<0.001)$. There were no significant intergroup differences in the improvement indices when $\alpha \leq 30^{\circ}$ (Table 2). ROC curve analysis, based on a grouping by an $\alpha$ of $10^{\circ}, 20^{\circ}$, and $30^{\circ}$, revealed that grouping by $30^{\circ}$ achieved the best AUC (0.828). Hence, grouping by $\alpha=30^{\circ}$ achieved excellent discrimination, while grouping by $\alpha=10^{\circ}(\mathrm{AUC}=0.532)$ or $\alpha=20^{\circ}$ (AUC $\left.=0.621\right)$ achieved poor discrimination. The DeLong test for the three ROC curves also revealed that the AUC, when grouped by $\alpha=30^{\circ}$, was significantly different from the AUCs when grouped by $\alpha=10^{\circ}$ or $\alpha=20^{\circ}$; however, there were no significant differences in the AUCs when grouped by $\alpha=10^{\circ}$ and $\alpha=20^{\circ}$ (Fig. 3). Univariate analysis revealed that $\alpha>30^{\circ}$ was associated with significantly higher body weight and higher $\mathrm{HI}$ values. However, there were no significant differences in age at repair, the number of bars, follow-up period, sex,
Table 2 Multiple comparisons between the four groups (Scheffe post hoc test)

\begin{tabular}{llll}
\hline (I) Group & (J) Group & Mean Dif $(\mathbf{I}-\mathbf{J})$ & $\boldsymbol{p}$-value \\
\hline$\leq 10^{\circ}$ & $11-20^{\circ}$ & -0.012 & 0.850 \\
& $21-30^{\circ}$ & 0.009 & 0.974 \\
& $>30^{\circ}$ & 0.136 & $<\mathbf{0 . 0 0 1}$ \\
& $\leq 10^{\circ}$ & 0.012 & 0.850 \\
$11-20^{\circ}$ & $21-30^{\circ}$ & 0.02 & 0.732 \\
& $>30^{\circ}$ & 0.148 & $<\mathbf{0 . 0 0 1}$ \\
& $\leq 10^{\circ}$ & -0.009 & 0.974 \\
$21-30^{\circ}$ & $11-20^{\circ}$ & -0.02 & 0.732 \\
& $>30^{\circ \dagger}$ & 0.127 & $<\mathbf{0 . 0 0 1}$
\end{tabular}

${ }^{\dagger}$ There is a statistical difference between group $>30^{\circ}$ and other groups $(p<0.001)$

$p<0.05$ are given in bold

scoliosis, or asymmetric body features between patients with $\alpha>30^{\circ}$ and with $\alpha \leq 30^{\circ}$ (Table 3). Furthermore, 7 of 23 patients in the $\alpha>30^{\circ}$ group voluntarily underwent a revision Nuss procedure after the comprehensive evaluation of significant recurrence and symptoms. These seven patients comprised five men and two women, and the mean age was $25 \pm 8.2$ years. The median [interquartile range (IQR)] period between the revision surgery and the first operation was 49 days (IQR: 27-71 days). Patients who underwent the revision Nuss procedure experienced a significantly greater improvement in the $\alpha$ and cxrHI values than after the first surgery $(p<0.05)$ (Table 4$)$.

\section{Discussion}

Minimally invasive repair of pectus excavatum (MIRPE), Nuss procedure, specially modified to be performed with a thoracoscopic aid [8] being used to correct pectus excavatum has shown satisfactory long-term results along with a better quality of life in over $90 \%$ cases who underwent primary repair, similar to the results of our clinical study $[6,14,15]$. The most common complication of the Nuss procedure is bar placement [16]. In our study, we used the lateral fixation method bilaterally with wire or stabilizers. The majority of the bar displacements in our cases were due to bar flipping, although we did encounter a few inconspicuous lateral migration and undetectable dorsal shift migration cases that could be disregarded. Hoksch et al. reported that in their analysis of 129 cases, 9 patients (7.0\%) required surgical revision due to bar displacement [15]. Kelly et al. which reported by far the biggest case analysis with 1215 patients who underwent minimally invasive repair, reported that $4 \%$ of their cases had postoperative complications of the displacement of bars which required surgical revision [6]. Nevertheless, $\mathrm{PE}$ recurrence remains the most important unfavorable 
Table 3 Comparison of the clinical characteristics based on a grouping by $30^{\circ}$

\begin{tabular}{|c|c|c|c|}
\hline & $a \leq 30^{\circ}(n=336)$ & $a>30^{\circ}(n=23)$ & $p$-value \\
\hline Age at Nuss repair, years (mean \pm SD) & $23.8 \pm 7.8$ & $25 \pm 6.8$ & 0.409 \\
\hline Sex, $n(\%)$ & & & 0.754 \\
\hline Male & $284(85 \%)$ & $20(87 \%)$ & \\
\hline Female & $52(15 \%)$ & $3(13 \%)$ & \\
\hline Flipping angle, degrees (mean $\pm S D$ ) & $12.8 \pm 6.3$ & $40.4 \pm 5.8$ & $<0.001$ \\
\hline Body weight, kg (mean \pm SD) & $57.6 \pm 10.7$ & $63.6 \pm 11.5$ & 0.01 \\
\hline Haller index, $($ mean $\pm S D)$ & $4 \pm 0.9$ & $4.4 \pm 1.8$ & 0.018 \\
\hline Observation period, years (mean \pm SD) & $2.2 \pm 1.3$ & $1.9 \pm 1.6$ & 0.405 \\
\hline $\operatorname{BarN}^{+}, n(\%)$ & & & 0.641 \\
\hline 1 & $33(10 \%)$ & $2(9 \%)$ & \\
\hline 2 & $269(80 \%)$ & $20(87 \%)$ & \\
\hline 3 & $34(10 \%)$ & $1(4 \%)$ & \\
\hline Scoliosis, n, (\%) & & & 0.596 \\
\hline Yes & $316(94 \%)$ & $21(91 \%)$ & \\
\hline No & $20(6 \%)$ & $2(9 \%)$ & \\
\hline Symmetry n, (\%) & & & 0.593 \\
\hline Yes & $156(46 \%)$ & $12(52 \%)$ & \\
\hline No & $180(54 \%)$ & $11(48 \%)$ & \\
\hline
\end{tabular}

${ }^{\dagger}$ BarN: the number of inserted bars in each patient

$p<0.05$ are given in bold

Table 4 Comparison of the slope angle and the Haller index in seven patients before and after the revision Nuss surgery

\begin{tabular}{cccc}
\hline & $\begin{array}{l}\text { Before revision } \\
\text { surgery }\end{array}$ & $\begin{array}{l}\text { After revision } \\
\text { surgery }\end{array}$ & p value \\
\hline $\begin{array}{c}\text { Slope angle } \\
\text { a (IQR), } \\
\text { degrees }\end{array}$ & $39.6(38.5-52)$ & $10.4(9.1-23.7)$ & $\mathbf{0 . 0 1 8}$ \\
cxrHI (IQR) & $3.5(3.1-3.8)$ & $2.9(2.5-3.1)$ & $\mathbf{0 . 0 4 3}$ \\
\hline
\end{tabular}

There was a significant difference $(p<0.05)$ in the $\mathrm{a}$ and $\mathrm{HI}$ before and after the revision Nuss procedure

$p<0.05$ are given in bold

outcome of the Nuss repair. Previous studies have reported that the risk factors for PE recurrence after the Nuss procedure include surgical complications, younger age, earlier bar removal, higher HI, and higher body weight [3-6]. Contrary to other study that focused on PE recurrence after bars removal, our study discusses PE recurrence before bar removal [12]. We believe that bar flipping affects PE recurrence to some extent; however, in our clinical observation on postoperative follow-up chest radiography, in some cases, there was no obvious chest wall recession despite signs of bar flipping. Few studies have defined the bar displacement, or proposed how the displacement affects the operation revision. Moreover, the degree of bar displacement and the method to measure it are still inconsistent and seldomly mentioned.
Kelly et al. reported that a bar movement more than fifteen degree is indicative for operative repositioning [6]. Cho et al. defined bar displacement more than twenty degree as severe bar migration [12]. However, in our study, no statistical differences in PE improvement were observed when the flipping of bars is less than $30^{\circ}$. We presumed that one of the reason causes the discrepancy of angle between the above studies and ours may be due to a difference in the method to determine bar flipping. Moreover, the authors of the above two studies have not set the reference point when mentioned the "bar movement". Furthermore, Cho et al. used only one arm of the bar to measure the flipping angle which may change due to slight changes in the shooting angle of the follow-up plain film and the author didn't mention how to measure when the bar inserted obliquely in certain cases. To diminish the deviation, we chose the middle point of the two arms as the reference point (Fig. 2). Similar to Cho's study, we also considered the optimal point of the dorm of the bar to be where the force vector application was perpendicular to the sternum. Different measurement tools, CT or plain film, may also influence the assessment of migration. We used a lateral view of chest radiography to determine the bar migration as well as PE recurrence by calculating the change in postoperative Haller index, as described in previous study $[11,12]$. They have indicated a significant correlation between Haller index using chest $\mathrm{CT}$ and simple radiographic data. 
In most of the reports, the recurrence of PE has been simply identified by re-cave-in appearance and patient's symptoms $[3-6,12]$. The distinction between our work and the others is that we did not consider bar migration and PE recurrence as "yes- or -no" events. We considered the bar's flipping and the degree of PE recurrence as "continuous variables". In our study, we used an improvement index to quantify PE recurrence. Since poor improvement in $\mathrm{PE}$ after surgery can indicate $\mathrm{PE}$ recurrence, we used $\Delta \mathrm{cxrHI}$, the change between preand postoperative cxrHI (i.e., the improvement index) to objectively estimate the degree of PE recurrence. A significant factor that influences the improvement index was a flipping angle of more than $30^{\circ}$. Sa et al. designed an original method to measure bar migration in which they calculated the distance from the sternal angle to the upper border of the metal bar on the lateral chest radiograph to represent the bar's migration. They had 61 patients who underwent the primary Nuss procedure, of whom 7 (11\%) required reoperation for bar displacement. By standardizing the distance of migration $\left(\mathrm{D}_{0}-\mathrm{Dx} /\right.$ $\left.\mathrm{D}_{0} \times 100\right)$, they developed a bar displacement index (BDI) and found that the optimal cut-off value of BDI warranting reoperation was 8.7 [17].

In our previous study, postoperative changes in chest wall shapes mostly occurred within one month, and the chest wall diameter stabilized three months after the Nuss repair [18]. All cases in our present study were followed up for at least six months, which was an adequate time period for monitoring PE recurrence. A correlation between bar flipping and PE recurrence can be explained as follows: The convex bars strut the depressed sternum, and the tendency of the anterior chest wall to rebound inwards causes bar flipping even before the chest wall can be remodeled. The bar still has some strength when the flip angle is less than thirty degree. A tilted bar lacks support, which may cause PE recurrence; therefore, reciprocal causation is suggested between bar flipping and PE recurrence. Our study shows that the bar number does not influence postoperative bar flipping or the $\mathrm{PE}$ recurrence rate. This may be because the number of bars placed is decided by an immediate intraoperative outcome which means we would insert additional bars if one did not have enough supporting strength for lifting the concave chest wall. Although twenty-three of our patients with bar flipping angle more than thirty degree had PE recurrence, only seven of them underwent a revision Nuss procedure after evaluating aspects such as the severity of recurrence and the patient's willingness and symptoms. For patients who underwent a revision repair for PE recurrence, the Nuss procedure remains an adequate choice with good outcomes, regardless of whether the initial repair was also a Nuss procedure; this is consistent with the results from Casamassima et al. [19] We hypothesize that the repositioning of the Nuss bars in a revision procedure increases the likelihood of successfully lifting the depressed anterior chest wall and provides additional time for chest wall remodeling.

We acknowledge that this study was retrospective and was performed at a single institute. Our case numbers with a bar flipping angle more than thirty degree and revision cases were limited. Researchers are encouraged to use our test model to conduct more clinical studies and provide solid evidence on PE recurrence. Moreover, a longer follow-up period is still required for patients who undergo a revision Nuss procedure.

\section{Conclusions}

$\mathrm{PE}$ recurrence is the biggest concern in patients undergoing the Nuss procedure. In this study, the patients comprised adults who underwent an initial surgical PE repair. We introduced a simple and effective method for determining bar flipping. Our findings indicate that a bar flipping angle more than thirty degree is a predictor of $\mathrm{PE}$ recurrence and may require revision surgery. Our study's limitations include its single-center, retrospective nature, and the fact that only one thoracic surgeon performed all surgeries. More cases are still required to support our findings. The Nuss procedure is an adequate follow-up intervention in patients with PE recurrence who underwent the Nuss procedure as the primary repair; however, a long-term follow-up is still required for such patients.

\section{Abbreviations \\ ANOVA: One-way analysis of variance; AUC: Area under the curve; $\mathrm{Cl}$ : Confidence interval; CT: Computed tomography; $\mathrm{CxrHI}$ : Chest $\mathrm{X}$-ray HI; HI: Haller index; IQR: Interquartile range; Postop cxrHI: Postoperative cxrHI; Preop cxrHI: Preoperative cxrHI; PE: Pectus excavatum; ROC: Receiver operating characteristics.}

\section{Acknowledgements \\ None.}

\section{Authors' contributions}

Conception and design: Y-LC; Administrative support: Y-LC, Y-YH, I-ST; Provision of study materials or patients: Y-LC, Y-YH; Collection and assembly of data: Y-JF, P-CL, B-CW, Y-LC; Data analysis and interpretation: Y-JF, I-ST, B-CW, Y-LC; Manuscript writing: Y-JF, Y-LC; Final approval of manuscript: all authors.

\section{Funding}

This work was supported by the Buddhist Tzu Chi Medical Foundation of the Taipei Tzu Chi Hospital [TCRD-TPE-110-21].

Availability of data and materials

The data sets used and analyzed during the current study are available from the corresponding author.

\section{Declarations}

Ethics approval and consent to participate

The authors are accountable for all aspects of the work in ensuring that questions related to the accuracy or integrity of any part of the work are 
appropriately investigated and resolved. The study was conducted in accordance with the Declaration of Helsinki (as revised in 2013). This study was approved by the Ethics Committee of and the Institutional Review Board of the Taipei Tzu Chi Hospital, Taipei, Taiwan, ROC (IRB No: 08-X-101), and individual consent for this retrospective analysis was waived.

\section{Consent for publication}

Informed consent was obtained from the patient for publication of chest X-ray images.

\section{Competing interests}

The authors declare that they have no competing interests.

\section{Author details}

'Division of Thoracic Surgery, Department of Surgery, Taipei Tzu Chi Hospital, Buddhist Tzu Chi Medical Foundation, New Taipei City, No. 289, Jianguo Road, Xindian District 231, Taiwan. ${ }^{2}$ Department of Radiology, Taipei Tzu Chi Hospital, Buddhist Tzu Chi Medical Foundation, New Taipei City, Taiwan. ${ }^{3}$ Department of Research, Taipei Tzu Chi Hospital, Buddhist Tzu Chi Medical Foundation, New Taipei City, Taiwan. ${ }^{4}$ School of Medicine, Tzu Chi University, Hualien, Taiwan.

Received: 27 January 2021 Accepted: 12 August 2021

Published online: 28 August 2021

\section{References}

1. Biavati M, Kozlitina J, Alder AC, Foglia R, McColl RW, Peshock RM, Kelly RE $\mathrm{Jr}$, Kim Garcia C. Prevalence of pectus excavatum in an adult populationbased cohort estimated from radiographic indices of chest wall shape. PLoS ONE. 2020;15(5):e0232575.

2. Cheng YL, Tzeng IS, Yang MC. Increased prevalence of obstructive sleep apnea in patients with pectus excavatum: a pilot study. Ci Ji Yi Xue Za Zhi. 2018;30(4):233-7.

3. Tikka T, Kalkat MS, Bishay E, Steyn RS, Rajesh PB, Naidu B. A 20-year review of pectus surgery: an analysis of factors predictive of recurrence and outcomes. Interact Cardiovasc Thorac Surg. 2016;23(6):908-13.

4. Pawlak K, Gasiorowski L, Gabryel P, Galecki B, Zielinski P, Dyszkiewicz $W$. Early and late results of the nuss procedure in surgical treatment of pectus excavatum in different age groups. Ann Thorac Surg. 2016;102(5):1711-6

5. Gibreel W, Zendejas B, Joyce D, Moir CR, Zarroug AE. Minimally invasive repairs of pectus excavatum: surgical outcomes, quality of life, and predictors of reoperation. J Am Coll Surg. 2016;222(3):245-52.

6. Kelly RE, Goretsky MJ, Obermeyer R, Kuhn MA, Redlinger R, Haney TS, Moskowitz A, Nuss D. Twenty-one years of experience with minimally invasive repair of pectus excavatum by the Nuss procedure in 1215 patients. Ann Surg. 2010;252(6):1072-81.

7. Binkovitz LE, Zendejas B, Moir CR, Binkovitz LA. Nuss bar migrations: occurrence and classification. Pediatr Radiol. 2016;46(13):1797-803.

8. Lo PC, Tzeng IS, Hsieh MS, Yang MC, Wei BC, Cheng YL: The Nuss procedure for pectus excavatum: An effective and safe approach using bilateral thoracoscopy and a selective approach to use multiple bars in 296 adolescent and adult patients. PLoS ONE 2020;15(5):e0233547.

9. Nuss D, Kelly RE Jr. Indications and technique of Nuss procedure for pectus excavatum. Thorac Surg Clin. 2010;20(4):583-97.

10. Haller JA Jr, Kramer SS, Lietman SA. Use of CT scans in selection of patients for pectus excavatum surgery: a preliminary report. J Pediatr Surg. 1987;22(10):904-6.

11. Wu TH, Huang TW, Hsu HH, Lee SC, Tzao C, Chang H, Cheng YL. Usefulness of chest images for the assessment of pectus excavatum before and after a Nuss repair in adults. Eur J Cardiothorac Surg. 2013;43(2):283-7.

12. Cho DG, Kim JJ, Park JK, Moon SW. Recurrence of pectus excavatum following the Nuss procedure. JThorac Dis. 2018;10(11):6201-10.

13. DeLong ER, DeLong DM, Clarke-Pearson DL. Comparing the areas under two or more correlated receiver operating characteristic curves: a nonparametric approach. Biometrics. 1988;44(3):837-45.

14. Yang MC, Lan CC, Wu YK, Hsieh MS, Wei BC, Cheng YL. Improvement in polysomnographic objective sleep quality in adults with pectus excavatum after the Nuss procedure. World J Surg. 2020;44(4):1316-22.

15. Hoksch B, Kocher G, Vollmar P, Praz F, Schmid RA. Nuss procedure for pectus excavatum in adults: long-term results in a prospective observational study. Eur J Cardiothorac Surg. 2016;50(5):934-9.

16. Goretsky MJ, McGuire MM. Complications associated with the minimally invasive repair of pectus excavatum. Semin Pediatr Surg. 2018;27(3):151-5.

17. Sa YJ, Lee J, Jeong JY, Choi M, Park SS, Sim SB, Jo KH. A clinical decisionmaking model for repeat surgical treatment of pectus Bar displacement: distance measurement after nuss procedure. J Cardiothorac Surg. 2016;11:16.

18. Yeh SW, Yang MC, Chang YW, Hsu YT, Tzeng IS, Cheng YL. Improved dynamics of thoracic cage and exercise capacity after Nuss repair for pectus excavatum. Thorac Cardiovasc Surg. 2020;68(1):85-91.

19. Sacco Casamassima MG, Papandria D, Goldstein SD, Yang J, Mclltrot KH, Abdullah F, Colombani PM. Contemporary management of recurrent pectus excavatum. J Pediatr Surg. 2015;50(10):1726-33.

\section{Publisher's Note}

Springer Nature remains neutral with regard to jurisdictional claims in published maps and institutional affiliations.

Ready to submit your research? Choose BMC and benefit from:

- fast, convenient online submission

- thorough peer review by experienced researchers in your field

- rapid publication on acceptance

- support for research data, including large and complex data types

- gold Open Access which fosters wider collaboration and increased citations

- maximum visibility for your research: over $100 \mathrm{M}$ website views per year

At BMC, research is always in progress.

Learn more biomedcentral.com/submissions 